\title{
Femtosecond Extreme Ultraviolet Ion Imaging of Ultrafast Dynamics in Electronically Excited Helium Nanodroplets
}

\author{
Oliver Bünermann, Oleg Kornilov, Stephen R. Leone, Daniel M. Neumark, and Oliver Gessner
}

(Invited Paper)

\begin{abstract}
A novel femtosecond extreme ultraviolet (EUV) ionimaging technique is applied to study ultrafast dynamics in electronically excited helium nanodroplets. Ion mass spectra recorded by single-photon EUV ionization and by transient EUV-pump/IRprobe two-photon ionization differ significantly for EUV photon energies below and above $\sim 24 \mathrm{eV}$, in agreement with recently performed synchrotron measurements. Pump-probe time-delaydependent ion kinetic energy (KE) spectra exhibit two major contributions: a decaying high $\mathrm{KE}$ component and a rising low KE component, which are attributed to the different excitation regimes. A model is presented that describes the excitation energy dependence of the relaxation and ionization dynamics within the framework of bulk and surface states. The model is supported by recent $a b$ initio calculations on electronically excited states of 25-atom clusters. An intraband relaxation mechanism is proposed that proceeds on a $\sim 10-20$-ps time scale and that corresponds to the transfer of electronic excitation in the Rydberg $n=2$ manifold from bulk to surface states.
\end{abstract}

Index Terms-Photochemistry, physics, photonics, UV sources.

\section{INTRODUCTION}

$\mathbf{T}$ HE ADVENT of femtosecond extreme ultraviolet (EUV) laser light sources has enabled a new class of time-domain

Manuscript received October 16, 2010; revised December 14, 2010; accepted January 21, 2011. Date of publication April 7, 2011; date of current version January 31, 2012. This work was supported by the Director, Office of Science, Office of Basic Energy Sciences, Chemical Sciences Division, U.S. Department of Energy under Contract DE-AC02-05CH1 1231. The tenure of O. Bünermann at the Ultrafast X-ray Science Laboratory was supported by a Research Fellowship from the Deutsche Forschungsgemeinschaft.

O. Bünermann was with the Ultrafast X-ray Science Laboratory, Chemical Sciences Division, Lawrence Berkeley National Laboratory, Berkeley, CA 94720 USA and the Department of Chemistry, University of California, Berkeley, CA 94720 USA. He is now with the Institute of Physical Chemistry, GeorgAugust-University Göttingen, 37077 Göttingen, Germany.

O. Kornilov was with the Ultrafast X-ray Science Laboratory, Chemical Sciences Division, Lawrence Berkeley National Laboratory, Berkeley, CA 94720 USA and the Department of Chemistry, University of California, Berkeley, CA 94720 USA. He is now with the Max-Born-Institute, Berlin 12489, Germany.

S. R. Leone is with the Ultrafast X-ray Science Laboratory, Chemical Sciences Division, Lawrence Berkeley National Laboratory, Berkeley, CA 94720 USA and the Departments of Chemistry and Physics, University of California, Berkeley, CA 94720 USA.

D. M. Neumark is with the Ultrafast X-ray Science Laboratory, Chemical Sciences Division, Lawrence Berkeley National Laboratory, Berkeley, CA 94720 USA and the Department of Chemistry, University of California, Berkeley, CA 94720 USA.

O. Gessner is with the Ultrafast X-ray Science Laboratory, Chemical Sciences Division, Lawrence Berkeley National Laboratory, Berkeley, CA 94720 USA (e-mail: ogessner@lbl.gov).

Color versions of one or more of the figures in this paper are available online at http://ieeexplore.ieee.org.

Digital Object Identifier 10.1109/JSTQE.2011.2109054 experiments. Laboratory-based high-order harmonic generation (HHG) driven by intense femtosecond infrared (IR) lasers is employed to study the evolution of UV-excited molecular states [1], [2], to disentangle the quantum state distribution in strong fieldionized systems [3], [4], and to monitor the ionization and relaxation dynamics of EUV-excited atoms and molecules [5]-[10]. Here, we combine a HHG light source with state-of-the-art 3-D momentum-resolved ion mass spectrometry to investigate the relaxation dynamics of electronically excited helium nanodroplets. A similar experimental approach facilitated femtosecond time-resolved EUV electron and ion-imaging experiments on diatomic molecules by the group of Kapteyn and Murnane [6], [7], [11]. However, to the best of our knowledge, this is the first time that such a method has been applied to disentangle ultrafast EUV-induced dynamics in a complex system comprising $\sim 2 \times 10^{6}$ atoms.

These droplets distinguish themselves from other noble gas clusters by their unique thermodynamic properties. Formed in a supersonic expansion through a cryogenically cooled nozzle, the droplets reach an equilibrium temperature of $0.37 \mathrm{~K}$ within milliseconds by means of an evaporative cooling mechanism [12]. At this temperature, helium is in its superfluid (He II) phase, which results in helium nanodroplets with a remarkable set of characteristics. They form, for example, an ideal matrix for high-resolution spectroscopy studies on molecular systems that comprise several dozens of atoms [13] and for the controlled assembly of clusters and molecules with structural properties that are not achieved in any other environment [14], [15]. Dopant species are rapidly cooled to the sub-Kelvin equilibrium temperature of pure droplets, while their physical properties are only very weakly perturbed by the superfluid environment. This capability has been beautifully demonstrated by the seminal experiments of Toennies et al. who recorded rotationally resolved IR absorption spectra of $\mathrm{SF}_{6}$ and carbonyl sulfide molecules residing in ${ }^{4} \mathrm{He}$ clusters comprising 60-6000 atoms [16]-[18]. Most recently, it has been demonstrated that sufficiently large droplets can host entire proteins in the size range of 12000 atomic mass units [19]. These findings are encouraging for plans to employ helium nanodroplets in experiments that will determine the structures of biomolecules that cannot be crystallized [20] and as vehicles for soft-landing surface deposition of nanostructures [14].

The excitement about the opportunities that arise in fundamental and applied sciences through the use of helium 


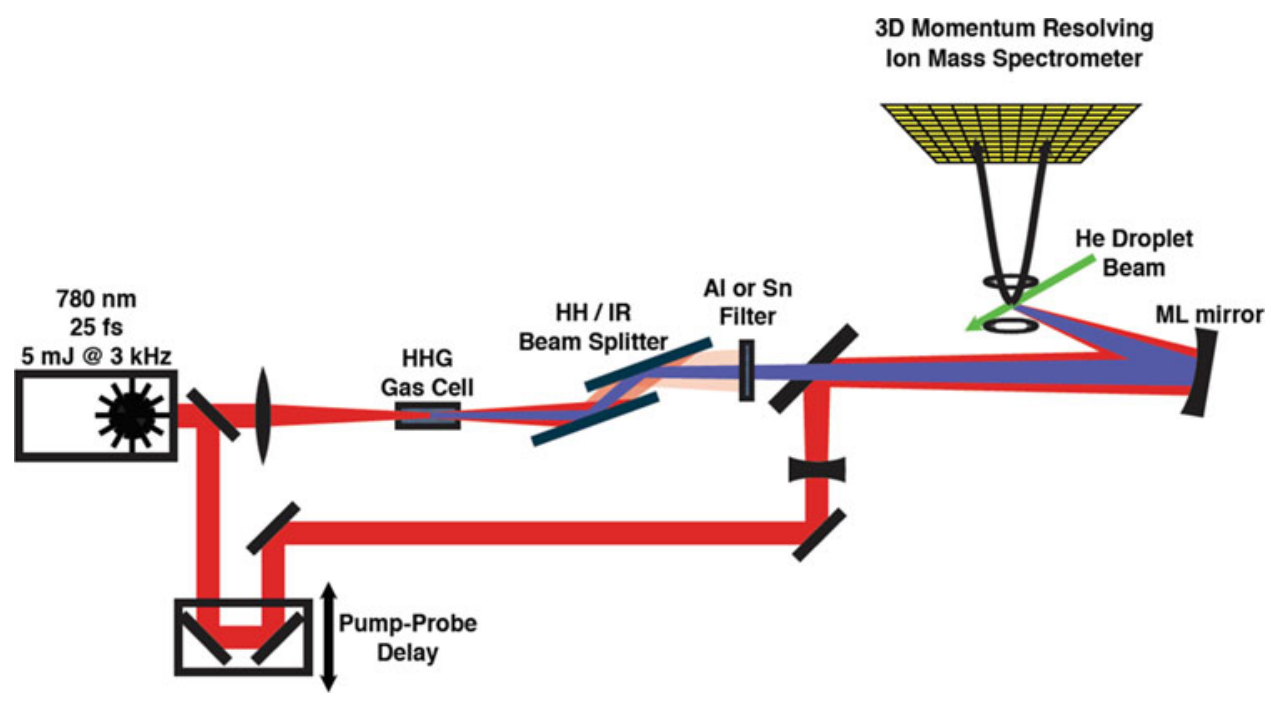

Fig. 1. Experimental setup for femtosecond time-resolved and 3-D-momentum-resolved EUV ion mass spectrometry.

nanodroplet matrices is contrasted by the limited knowledge of their fundamental physical properties. Since Möller et al. recorded the EUV absorption spectrum of helium clusters in the early 1990s [21], little progress has been made in understanding the nature of the two major electronic bands at excitation energies of $\sim 21.6$ and $\sim 23.8 \mathrm{eV}$. The droplet relaxation dynamics after electronic excitation are even more poorly understood. Synchrotron-based energy-resolved EUV fluorescence measurements of the Möller group identify the emission of electronically excited helium atoms and helium dimers as an important de-excitation mechanism [22]. Photoionization and photoelectron imaging studies in the groups of Toennies and Neumark provide evidence for an indirect ionization process at excitation energies beyond $\sim 23 \mathrm{eV}$ [23], [24].

Most recently, Kornilov et al. employed femtosecond timeresolved EUV-pump/IR-probe photoelectron imaging spectroscopy to directly monitor the de-excitation dynamics of large, electronically excited helium nanodroplets in the time domain [25]. This study provides evidence for initial rapid relaxation on a time scale of $0.2-0.3$ ps along with efficient interband relaxation from the upper $(\sim 23.8 \mathrm{eV})$ to the lower $(\sim 21.6 \mathrm{eV})$ electronic band of the droplet on a time scale of 2-3 ps. The physical nature of the transiently populated states, however, and the pathway of the indirect droplet ionization process remain elusive. A unique identification of the relaxation dynamics is in part hampered by uncertainty about the nature of the ionized species. From the photoelectron spectrum alone, it is not possible to uniquely associate every observed dynamics channel with probe-pulse ionization of the initially excited droplet, a partially relaxed droplet, or excited cluster fragments. The transient ion-imaging study presented here is motivated by the expectation that different ionization channels lead to different ion mass spectra and different kinetic energies of the emitted ions.

The measurements are performed using two different excitation spectra defined by the transmission functions of an aluminum filter and a tin filter, respectively. Pump-probe timedelay-dependent (i.e., transient) ion kinetic energy (KE) dis- tributions facilitate the distinction of decaying and rising ion yield contributions, thus providing complementary information to the photoelectron experiments. The ion mass spectra differ dramatically for experiments with the Al filter and the Sn filter, respectively. The dynamics of the partial ion yields recorded with the Al filter depend critically on the ion mass. The different dynamic trends are explained in a model that distinguishes between bulk and surface excitations. The model is supported by recent ab initio calculations of Closser and Head-Gordon [26]. An intraband relaxation mechanism in the lower electronic band of the droplets at $\sim 21.6 \mathrm{eV}$ is proposed that corresponds to the transfer of electronic excitation in the Rydberg $n=2$ manifold from bulk to surface states on a time scale of $\sim 10-20 \mathrm{ps}$.

\section{EXPERIMENT}

Fig. 1 shows the experimental setup. A detailed description of the laser system, the high-harmonic generation setup and the optical routing has been given earlier [25]. Briefly, a femtosecond EUV light source based on HHG is interfaced with a molecular beam end-station that produces helium nanodroplets. Femtosecond EUV pulses (20 pJ, pump beam) are generated by focusing intense femtosecond IR $(785 \mathrm{~nm})$ laser pulses into a gas cell filled with krypton at a stagnation pressure of 2.5 torr. The copropagating fundamental IR light is removed from the EUV beam path by a two-stage silicon dichroic mirror [27] followed by either a $100-n m-t h i c k$ aluminum filter or a $200-\mathrm{nm}$ thick tin filter. In a second beam branch, a small fraction of the IR laser light (probe beam) with a pulse energy of $\sim 1 \mu \mathrm{J}$ (at the interaction volume) is routed through a retroreflector mounted on a computer-controlled translation stage, allowing for the adjustment of the relative time delay between the EUV and the IR pulses. The IR beam is recombined with the EUV beam by means of an annular mirror, positioned at a $45^{\circ}$ angle relative to the EUV beam propagation direction. The EUV beam passes through the center hole of the mirror, while the IR beam is reflected off the mirror in a donut-shaped spatial mode. A curved 


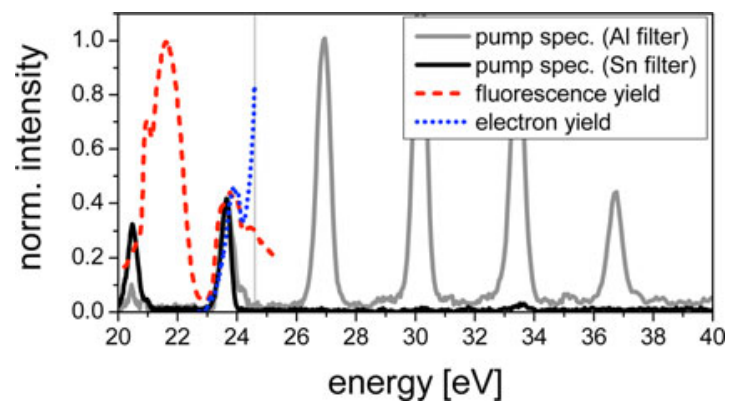

Fig. 2. EUV pump spectra filtered by an $\mathrm{Al}$ foil (gray) and an Sn foil (black). Spectra were recorded before reflection off the ML mirror. The spectra are compared to the EUV fluorescence excitation spectrum of Joppien et al. (red, dashed) and the energy dependent total ionization yield of Peterka et al. (blue, dotted). The vertical bar marks the IP of atomic helium.

multilayer (ML) mirror focuses both beams onto the gas target. The overlap of the two focal volumes is optimized by adjusting the position of a concave lens in the IR beam path upstream from the beam combiner, establishing the same effective focal length for both beams.

$\mathrm{The} \mathrm{Mg} / \mathrm{SiC}$ multilayer structure of the focusing mirror is optimized to reflect at $h \nu=23.7 \mathrm{eV}$ (15th harmonic) and to suppress other EUV components in the vicinity of this photon energy. However, because of the finite bandwidth and periodic spacing of multiple maxima in the ML mirror reflectivity curve, multiple higher harmonics $(17$ th, 19th, ...) are also reflected, albeit with reduced efficiency. Additionally, lower harmonics are reflected due to the finite specular reflectivity of SiC below $24 \mathrm{eV}$ [28]. Due to the multiband reflectivity characteristic of the ML mirror, the filter material used in the EUV beam path has a major impact on the EUV spectrum that is incident on the target gas. The transmission of the aluminum filter in the photon energy range $15-70 \mathrm{eV}$ is $\sim 80 \%$, leading to contributions from many harmonics in the pump step of the experiment. In contrast, the tin filter has a narrow transmission window between $\sim 14 \mathrm{eV}$ and $\sim 24.5 \mathrm{eV}$ with a theoretical maximum of $\sim 23 \%$ transmission at $21 \mathrm{eV}$ and $\sim 10 \%$ transmission at $23.7 \mathrm{eV}$.

Fig. 2 shows sections of two high-order harmonic spectra within the spectral region of interest recorded with a home-built spectrometer [29] before the EUV beam is reflected by the ML mirror. The gray and black solid curves are measured using the Al filter and Sn filter, respectively, in the EUV beam path. The fluorescence excitation spectrum of Joppien et al. (red, dashed, $>10^{6}$ atoms/cluster [21]) and the photoelectron yield spectrum of Peterka et al. (blue, dotted, $\sim 10^{4}$ atoms/cluster [24]), both recorded with tunable synchrotron radiation light sources, are shown for comparison. The Sn filter transmission curve effectively eliminates pump beam contributions with photon energies $>24 \mathrm{eV}$. The difference in the intensity of the 13th harmonic $(\sim 20.5 \mathrm{eV})$ is caused by the filter materials and slightly different HHG conditions in the two measurements. Due to the aforedescribed effects, reflection off the ML mirror is expected to modify the peak ratios of the EUV pump beam spectra in a noticeable way, but the general characteristics remain the same.
In particular, strong contributions above $24 \mathrm{eV}$ photon energy in the Al-filtered spectrum distinguish it markedly from the Sn-filtered pump beam spectrum.

The helium droplet apparatus is described in detail elsewhere [30]. Briefly, helium droplets are generated by expanding helium gas (Airgas, 99.9999\% purity) at temperatures of $10-20 \mathrm{~K}$ and pressures of 20-80 bar through a 5- $\mu \mathrm{m}$ orifice into a vacuum chamber. After expansion and droplet formation, the beam passes through two skimmers with 2-mm orifice diameters before entering the interaction volume. For the experiments presented here, the nozzle stagnation pressure and temperature are set to $80 \mathrm{bar}$ and $13 \mathrm{~K}$, respectively. Under these conditions, the average droplet size is expected to be $\sim 2 \times 10^{6}$ atoms/cluster [12].

In order to record transient ion yields and transient ion KE distributions, the helium droplet apparatus is equipped with an ion spectrometer with imaging capabilities (see Fig. 1). The spectrometer comprises a standard Wiley-McLaren ion timeof-flight (TOF) setup [31] combined with a microchannel plate (MCP) equipped delay-line detector [32]. The active area of the detector has a diameter of $\sim 80 \mathrm{~mm}$. The spectrometer consists of four sections: two 10-mm-long acceleration regions, a 285-mm-long field-free drift tube, and a 10-mm-long additional acceleration region in front of the detector. The electric potentials on the spectrometer electrodes are set to $0 \mathrm{~V}$ (ion repeller), $-100 \mathrm{~V}$ (ion extractor), $-300 \mathrm{~V}$ (drift tube entrance and exit), and $-2500 \mathrm{~V}$ (front of the MCP). Two deflector plates mounted parallel to the spectrometer axis inside the drift tube may be used to deflect the ion trajectories along the gas beam propagation direction. This facilitates the detection of large cluster ions that would otherwise miss the detector due to the combined effect of their long TOFs and the longitudinal velocity of the gas beam.

The ion spectrometer is operated in counting mode, i.e., the TOF and the detector hit position of every single detected ion are stored for post-processing during the data analysis. From these data, the complete 3-D momentum vector of every single massselected ion at the moment of its emission from the cluster can be reconstructed. This enables the femtosecond time-resolved determination of EUV-pump/IR-probe-induced ion kinetics, such as ion momentum distributions, ion KE distributions, and ionemission angular distributions [11], [32]. Typical benchmark characteristics of the setup are a mass resolution of $m / \Delta m \sim 500$ and a resolving power of $E / \Delta E \sim 100$.

For each pump-probe time-delay, two datasets are recorded: one with only the pump beam incident on the target ("pumponly") and immediately afterward, a second set with both pump and probe beams incident on the target ("pump + probe"). All pump-probe data presented here are corrected for pump-only contributions by subtracting the pump-only intensities from the pump + probe signals. In order to minimize the influence of systematic experimental drifts on the measured transients, multiple pump-probe time-delay scans with opposite scan directions are averaged. The functionality of the ion-imaging setup and the associated calibration procedures are tested by measuring the well-known momentum of $\mathrm{He}^{+}$ions due to the mean velocity of an atomic helium beam over a wide range of nozzle temperatures. 


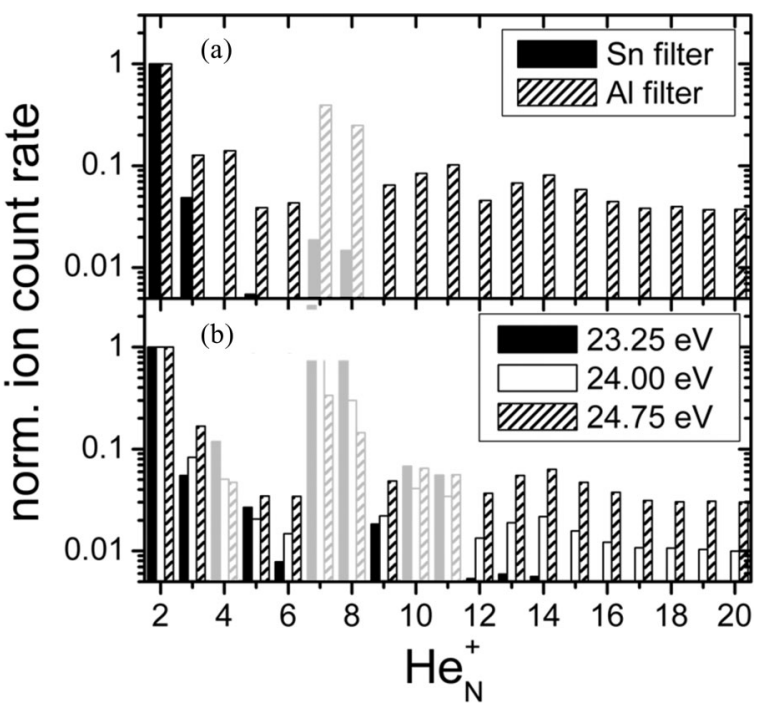

Fig. 3. (a) Single-photon mass spectra of $\mathrm{He}_{N}$ droplets $\left(\sim 2 \times 10^{6}\right.$ atoms/ cluster) recorded with EUV pump beams filtered by an Sn foil (solid) and an Al foil (striped). (b) Single-photon mass spectra of $\mathrm{He}_{N}$ droplets ( $\sim 10^{4}$ atoms/cluster) recorded at the Advanced Light Source with photon energies of $23.25 \mathrm{eV}$ (solid), $24.00 \mathrm{eV}$ (open), and $24.75 \mathrm{eV}$ (striped), respectively. The partly transparent mass peaks are contaminated by signals from residual gas in the experimental chamber.

\section{RESULTS}

\section{A. Single-Photon Ion Mass Spectra}

Fig. 3(a) shows two mass spectra of He droplets $(\sim 2 \times$ $10^{6}$ atoms/cluster) generated by single-photon ionization with the pump beam only (no probe beam). The solid and striped features are recorded using the Sn filter and the Al filter, respectively. The Al filter mass spectrum is marked by an abundance of large mass peaks that are absent in the Sn filter spectrum. The pump beam spectrum as defined by the different filter materials obviously has a crucial impact on the cluster ion distribution. For comparison, Fig. 3(b) shows mass spectra of He droplets (nozzle pressure/temperature: $60 \mathrm{bar} / 16 \mathrm{~K}, \sim 10^{4}$ atoms/cluster) recorded at the Chemical Dynamics Beamline of the Advanced Light Source at photon energies of $23.25 \mathrm{eV}$ (solid), $24.00 \mathrm{eV}$ (open), and $24.75 \mathrm{eV}$ (striped) [33]. All spectra in Fig. 3 are normalized to the same intensity of the $\mathrm{He}_{2}^{+}$peak, which is set to 1 . Note the logarithmic scale of the ordinate. Mass peaks that are contaminated by signals from residual gas in the experimental chamber are plotted as partly transparent. The monomer ion mass peak is not included, since it contains contributions from atomic helium background gas.

The photon-energy-dependent trend in the synchrotron mass spectra is illustrated in more detail in Fig. 4, which shows the ratio of the sum of all $\mathrm{He}_{N}^{+}$intensities with $N=12,13, \ldots, 20$ to the $\mathrm{He}_{2}^{+}$peak. The ratio rises by a factor of $\sim 20-50$ between 23.25 and $24.75 \mathrm{eV}$ and stays constant within the precision of the experiment between 24.75 and $25.50 \mathrm{eV}$.

\section{B. Pump-Probe Ion Mass Spectra}

Fig. 5(a) and (b) shows pump-probe-induced mass spectra measured with the Al filter and the Sn filter, respectively. Rep-

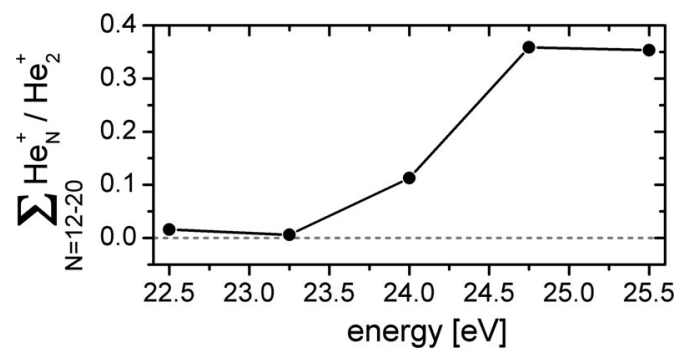

Fig. 4. Photon-energy-dependent ratio of large-to-small cluster fragments. The solid circles show the ratio of the sum of all $\mathrm{He}_{N}^{+}$intensities with $N=12$, $13, \ldots, 20$ to the dimer ion signal. The circles are connected by lines to guide the eye.

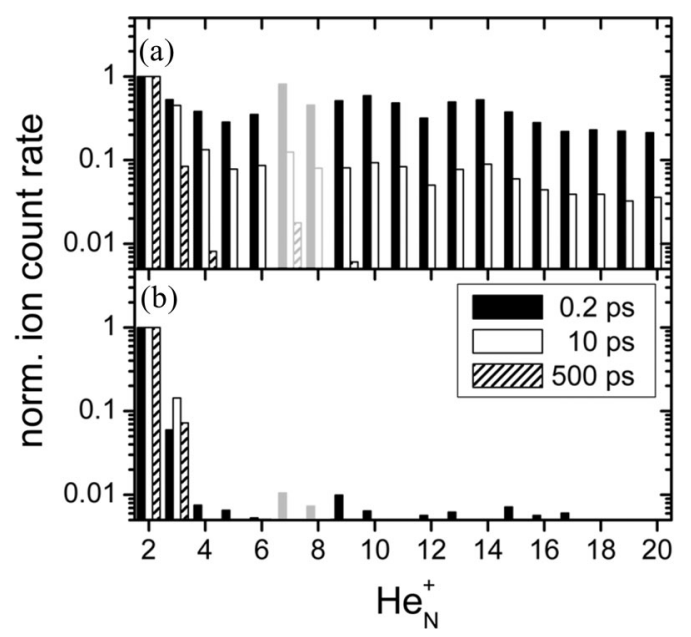

Fig. 5. EUV-pump/IR-probe mass spectra recorded at pump-probe time delays of $0.2 \mathrm{ps}$ (solid), $10 \mathrm{ps}$ (open), and $500 \mathrm{ps}$ (striped) for (a) Al-filtered and (b) Sn-filtered pump beam. The partly transparent mass peaks are contaminated by signals from residual gas in the experimental chamber.

resentative spectra are shown for pump-probe time delays of 0.2 ps (solid), 10 ps (open), and 500 ps (striped). All mass spectra are normalized to a dimer ion intensity of 1 . Due to the pump-only subtraction procedure, the intensities shown in Fig. 5 do not include signals from one-photon ionization. The probe-induced fraction of change in the $\mathrm{He}_{2}{ }^{+}$signal [(pump + probe)-pump-only)]/(pump-only) for pump-probe time delays of $0.2 / 10 / 500 \mathrm{ps}$ is $0.37 / 0.67 / 0.76$ for the Al filter data [see Fig. 5(a)] and 3.63/3.13/3.88 for the Sn filter data [see Fig. 5(b)], respectively.

The pump-probe spectra for $\mathrm{Al}$ and $\mathrm{Sn}$ filters at short time delays resemble the respective one-photon spectra in Fig. 3(a). For long time delays, however, the Al mass spectrum resembles that obtained with the Sn filter in that large mass peaks disappear. In the following, extensive datasets for multiple pump-probe time delays recorded with the Al filter in the EUV pump beam are presented. Extensive time-dependent Sn filter datasets will be the subject of a future publication.

\section{Transient Ion Yields and Kinetic Energy Distributions}

Fig. 6 shows the partial ion yields versus pump-probe delay of $\mathrm{He}_{N}^{+}$ions with $N=2,3,5,7$, and 12, normalized to the same maximum intensity at $\sim 0.2$ ps time delay. The dynamics for all 


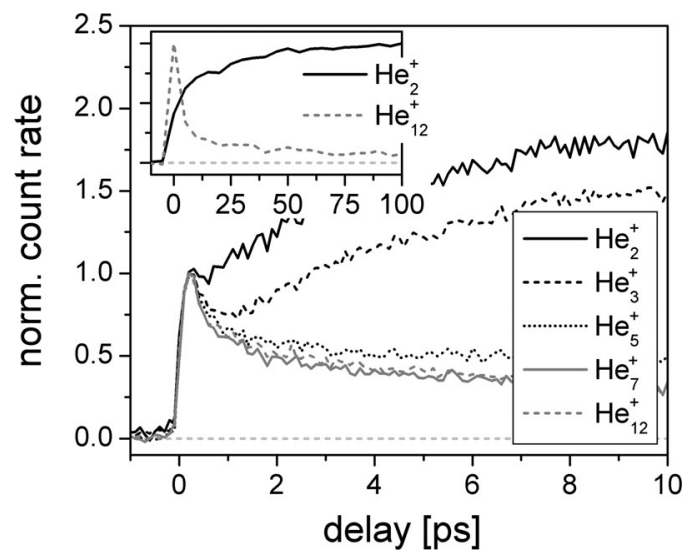

Fig. 6. Transient partial ion yields of different $\mathrm{He}_{N}^{+}$ions, normalized to the same maximum intensity at $\sim 0.2 \mathrm{ps}$. The inset shows the curves for $\mathrm{He}_{2}^{+}$(solid, black) and $\mathrm{He}_{12}^{+}$(dashed, gray) for pump-probe time delays up to $100 \mathrm{ps,}$ normalized to the same maximum intensity. The data were recorded with the $\mathrm{Al}$ filter in the EUV pump beam.

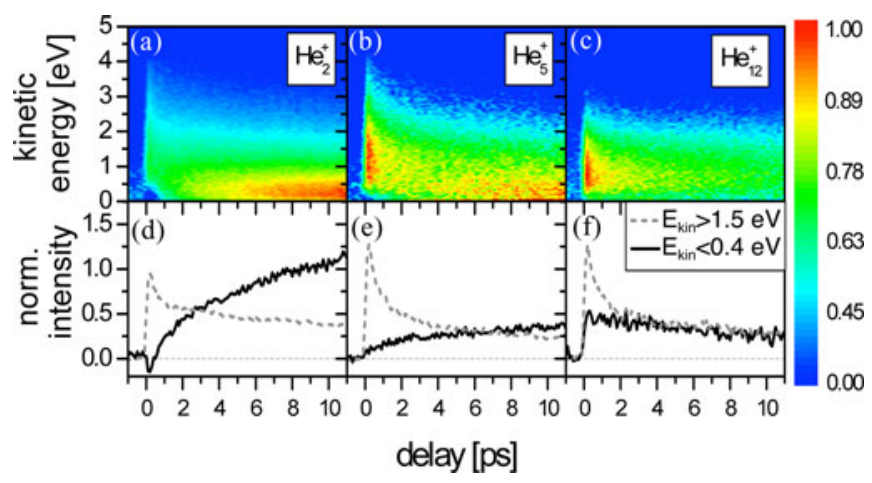

Fig. 7. (a)-(c) Transient ion $\mathrm{KE}$ distributions for $\mathrm{He}_{2}^{+}, \mathrm{He}_{5}^{+}$, and $\mathrm{He}_{12}^{+}$, respectively. The transients [(d)-(f)] are derived by integrating (a)-(c), respectively, over KE ranges of $0-0.4 \mathrm{eV}$ (black, solid) and $1.5-5 \mathrm{eV}$ (gray, dashed). The data are recorded with the $\mathrm{Al}$ filter in the EUV pump beam.

masses $N<7$ differ significantly, while the transient behavior of all masses with $N \geq 7$ is very similar as exemplified by the dashed gray curve $(N=12)$. The inset of Fig. 6 shows the pump-probe time-delay-dependent intensities of $\mathrm{He}_{2}^{+}$(solid, black) and $\mathrm{He}_{12}^{+}$(dashed, gray) ions over an extended delay range up to $100 \mathrm{ps}$. The $\mathrm{He}_{12}^{+}$transient is representative of the evolution of the Al filter spectrum from a broad mass distribution to a truncated spectrum that is very similar to the Sn filter spectrum. Comparison of the $\mathrm{He}_{2}^{+}$and $\mathrm{He}_{12}^{+}$curves shows that the long-term rise of the former and the long-term decay of the latter proceed on the same time scale of $\sim 10-20 \mathrm{ps}$, thus suggesting that the underlying dynamics may be linked by a common physical process.

Employing the 3-D-momentum resolution of the experimental setup, the transient partial ion yields are energy dispersed as presented in Fig. 7. Fig. 7(a)-(c) shows the pump-probe timedelay-dependent $\mathrm{KE}$ distributions of $\mathrm{He}_{2}^{+}, \mathrm{He}_{5}^{+}$, and $\mathrm{He}_{12}^{+}$ions, respectively. The curves in Fig. 7(d)-(f), are derived by integrating Fig. 7(a)-(c), respectively, over all KEs above $1.5 \mathrm{eV}$ (gray, dashed) and below $0.4 \mathrm{eV}$ (black, solid).

Comparison of Figs. 6 and 7 clearly demonstrates the gain in information that is available through the momentum imaging capability of the mass spectrometer. The decaying component in the $\mathrm{He}_{2}^{+}$yield that is easily identified in Fig. 7(a) and (d) cannot be discerned in the solid black curve of Fig. 6. Conversely, the rising component of Fig. 7(b) and (e) cannot be recognized in the $\mathrm{He}_{5}^{+}$yield of Fig. 6. Evidently, the 2-D transient ion KE maps of Fig. 7 facilitate the identification of different processes that are hard to distinguish in energy-integrated measurements.

From Fig. 7, it is evident that the transient ion signals shown in Fig. 6 comprise a rising and a decaying contribution, both of which are associated with different ion KE ranges. While the KEs of the decaying signal extend up to $\sim 5 \mathrm{eV}$, the rising signal seems to be confined to KEs below $\sim 1 \mathrm{eV}$. All high KE curves exhibit a characteristic instant rise near zero pump-probe delay followed by a monotonic decay that is marked by three time scales on the order of $\sim 0.2-0.3 \mathrm{ps}, \sim 2-3 \mathrm{ps}$, and $>10 \mathrm{ps}$. The two low KE curves in Fig. 7(d) and (e), in contrast, rise on a ps time scale starting from zero pump-probe delay. Only the black low KE curve in Fig. 7(f) seems to break the general pattern by exhibiting an instant rise followed by a slight decay. However, the reason for this behavior is evident from Fig. 7(c), which shows that the rising low-energy component is simply missing in the transient signal of the large $\mathrm{He}_{12}^{+}$cluster ions. A tail of the decaying high-energy component extends into the low KE range leading to the black trace in Fig. 7(f).

\section{DISCUSSION}

\section{A. Impact of Excitation Energies on Droplet Ionization Dynamics}

Mass spectra taken using femtosecond HHG and synchrotron radiation in Fig. 3 show that the ratio of the large ion signal $(N>3)$ to the smaller ion signal critically depends on the excitation photon energy. While excitation below $24 \mathrm{eV}$ (Sn-filtered EUV spectrum) leads almost exclusively to $\mathrm{He}_{N}^{+}$fragments with $N \leq 3$, ionization with higher photon energies leads to significant contributions from higher masses. Fig. 4 suggests that the transition between the two regimes proceeds continuously across the energy range from 23.25 to $24.75 \mathrm{eV}$. The smooth evolution of the mass spectrum is supported by the data of Fröchtenicht et al. [23]. The peak ratios of their threshold photoelectron photoion coincidence (TEPICO) spectrum recorded at $24.2 \mathrm{eV}$ are located between the extreme ratios displayed in Fig. 4. A continuous transformation of the ionization behavior below the ionization potential (IP) of atomic helium $(24.6 \mathrm{eV})$ is somewhat surprising. The photoelectron spectra of Peterka et al. do not exhibit any changes across the photon energy range starting from the droplet IP at $\sim 23 \mathrm{eV}$ to the IP of atomic helium [24]. However, the photon energy dependence of the total electron yield in [24] exhibits a rise with increasing photon energy starting at $\sim 24.2 \mathrm{eV}$ that cannot be explained by the onset of atomic helium ionization alone. Both the excellent energy resolution of the Chemical Dynamics Beamline cited in this study and the comparison with the atomic excitation spectra in [24] suggest that the rise of the electron yield beyond $24.2 \mathrm{eV}$ is, to a significant extent, the result of a change in the droplet ionization behavior. In experiments performed here with the $\mathrm{Al}$ filter, light 
above this energy can arise from the high energy side of the 15th harmonic or from higher harmonics.

We conclude that the ionization dynamics of helium droplets with more than $\sim 10^{4}$ atoms/cluster change markedly with excitation energy throughout the photon energy range that extends from the droplet IP at $\sim 23.0 \mathrm{eV}$ to the atomic helium IP at $24.6 \mathrm{eV}$. In particular, above $\sim 24.0-24.2 \mathrm{eV}$, the total ionization yield and the production of $\mathrm{He}_{N}^{+}$clusters with $N \geq 12$ increase rapidly toward higher photon energies. The photoelectron spectrum, however, is virtually unaffected by changes in the excitation energy (below the atomic IP), which severely limits the amount of information that can be gained on the varying ionization dynamics from static single-photon measurements.

The dramatic differences between the pump-probe timedelay-dependent mass spectra recorded with $\mathrm{Al}$ and $\mathrm{Sn}$ filters (see Fig. 5) indicate that information on the varying relaxation dynamics that result in the excitation-energy-dependent ion formation is contained in the transient ion data. Large fragments $(N>5)$, which only appear in relevant intensities for excitation above $\sim 24 \mathrm{eV}$, exhibit a monotonic decay that proceeds on three time scales (see Figs. 6 and 7), eventually leading to vanishing signal intensities by time delays of several hundred picoseconds [see Fig. 5(a) and Fig. 6 (inset)]. From Figs. 6 and 7 , it is evident that the signal decay is the only dynamic channel observed for these ions. Although the transient ion signals of the smaller fragments contain an additional rising component, the decaying component is also clearly visible in the transient energy maps [see Fig. 7(a), (b), (d), and (e)]. Apart from a slight shift to higher KEs and some broadening in the KE distribution, this component closely resembles the transient ion signal of the higher masses. This resemblance suggests that the decaying signal components in the transients of the smaller ions have predominantly the same origin as the dynamics observed for the higher masses, namely, excitation with photon energies above $\sim 24 \mathrm{eV}$. This inference is supported by the fact that the decaying component extends to very large KEs that require a pump photon energy beyond $24 \mathrm{eV}$ in order to fulfill energy conservation between the deposited photon energy and the potential and kinetic energies of the products (ion + photoelectron). We conclude that the decaying component of all ion transients is dominated by dynamics that are induced by droplet excitation beyond $\sim 24 \mathrm{eV}$. Conversely, excitation below $\sim 24 \mathrm{eV}$ in the pump-probe scheme leads predominantly to a rise of masses $N \leq 3$ and produces virtually no larger masses. Excitation above $\sim 24 \mathrm{eV}$, however, may also contribute to the rising components of the mass spectrum.

\section{B. Direct Versus Indirect Ionization Channels}

Fig. 8(a) shows a comparison of the transient ultraslow (ZEKE) photoelectron signal from Kornilov et al. (gray, dashed [25]) and the rising dimer ion signal from Fig. 7(d) (solid, black). Fig. 8(b) compares the transient photoelectron signal of Kornilov et al. that is associated with an isotropically emitted broad photoelectron band ( 0.7-1.1 eV KE, gray, dashed) and the high-KE ion signal of the $\mathrm{He}_{12}^{+}$fragments in Fig. 7(f). The similarities between the electron and ion curves in Fig. 8 suggest that the transients may be dominated by similar physical

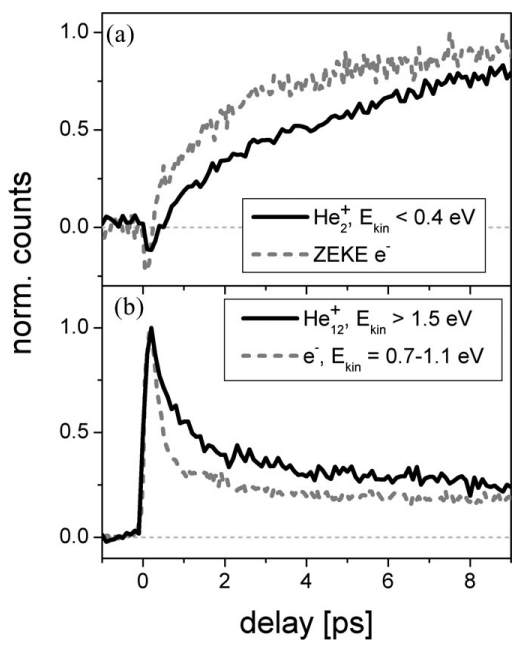

Fig. 8. Comparison of ion (black, solid) and electron (gray, dashed) transient signals. See text for details.

processes. The differences between the electron and ion curves in Fig. 8, on the other hand, indicate that there is no one-to-one correspondence between the respective electron and ion signals, and that both types of measurements provide complementary information. The curves in Fig. 8 can be approximated by either one or the sum of two exponentially rising/decaying contributions with time scales on the order of $0.14 \mathrm{ps}, 2.5 \mathrm{ps}$ for electrons and 12 ps for ions in Fig. 8(a); in Fig. 8(b), these are 0.28 ps, $2.8 \mathrm{ps}$ for electrons, and $0.7 \mathrm{ps}$ and $18 \mathrm{ps}$ for ions.

The comparison of the photoelectron and ion data presented in Fig. 8 suggests that the decaying ion signal component is mainly associated with direct ionization of excited droplet states by the probe pulse, and the rising ion signal component is mainly associated with the re-excitation mechanism that is proposed in [25]. In the re-excitation model, the probe pulse does not ionize the droplet directly, but it excites a neutral, partially relaxed droplet from the lower electronic band, centered around $\sim 21.6 \mathrm{eV}$, into the upper band, centered around $\sim 23.8 \mathrm{eV}$, which enhances the overall probability for indirect ionization and, therefore, the production of ultraslow photoelectrons.

The ZEKE electron signal in Fig. 8(a) rises faster during the first $\sim 10-20$ ps after excitation than the rising component of the $\mathrm{He}_{2}^{+}$signal. This suggests that indirect ionization by the probe pulse within $\sim 10-20 \mathrm{ps}$ after droplet excitation leads to other ions in addition to $\mathrm{He}_{2}^{+}$, and that the ratio of the yields for different ions that result from indirect ionization varies with pump-probe time delay. If the ratio of all ion yields from indirect ionization would be constant, all these partial ion yields would exhibit the exact same transient behavior as the ZEKE electrons. Energy conservation does not allow for the generation of monomer ions in the indirect ionization scheme. We conclude that ions larger than $\mathrm{He}_{2}^{+}$must be generated by indirect probe-pulse ionization during the first $\sim 10-20 \mathrm{ps}$, and that the yield of these larger ions must decline with increasing pump-probe delay compared to the yield of $\mathrm{He}_{2}^{+}$ions produced by indirect ionization. This conclusion is corroborated by the rising component in the $\mathrm{He}_{5}^{+}$yield [see Fig. 7(b) and (e)] and the asymptotic yields of all ions in Figs. 5 and 6, in particular, the 
inset in Fig. 6. The conclusion is also consistent with the slower decay of the $\mathrm{He}_{12}^{+}$signal compared to the electron signal from direct probe-pulse ionization in Fig. 8(b) provided that part of the $\mathrm{He}_{12}^{+}$signal stems from indirect probe-pulse ionization and that this component decays on a $\sim 10-20$-ps time scale.

\section{Physical Picture}

The excitation and relaxation dynamics of helium droplets are commonly discussed in terms of perturbed atomic and molecular states [22], [24], [34]-[42]. We note that, in particular, recent calculations by Closser and Head-Gordon indicate that this is an oversimplification [26]. Nevertheless, concentrating on excited states of helium atoms and dimers provides a good starting point for a qualitative description of the physics that underlie the results presented here.

The transition energy of $\sim 24 \mathrm{eV}$ for the onset of a pronounced large ion production is $\sim 1 \mathrm{eV}$ above the IP of large droplets and also above the energy of the lowest singlet excited atomic state $(1 \mathrm{~s} 3 \mathrm{p}, 23.1 \mathrm{eV})$ that is associated with the electronic band centered around $\sim 23.8 \mathrm{eV}$. It is known that the energies of excited atomic states are blue shifted by $\sim 1-1.6 \mathrm{eV}$ in a bulk liquid helium environment and in large droplets [43]-[46]. About the same energy is needed to inject an electron into bulk liquid helium, and strong indications for the onset of a $\sim 1-\mathrm{eV}$ barrier for photoemission from within large helium droplets ( $\sim 250000$ atoms/cluster) have been observed by Wang et al. [47]. This barrier is sometimes interpreted in terms of a conduction band edge [44], [46] in the sense that electrons with energies beyond this barrier may propagate unperturbed inside the droplet, while the motion of electrons with less energy is more restrained.

The differences in the dynamic trends observed for excitation above and below $24 \mathrm{eV}$ may be associated with the onset of excitation into perturbed, localized atomic states and/or the possibility for an electron to reach the conduction band of large droplets. In the latter case, however, a change in the photoelectron spectrum would be expected at the transition energy between a strongly hampered and a weakly perturbed propagation regime for electrons inside the cluster, but this is not the case [24]. The model of an onset of a perturbed atomic state excitation, in contrast, opens an interesting possibility to explain the different ionic products. Large clusters are known to have a $\sim 6$ - $\AA$-thick surface region across which the density of helium atoms rises from the free gas limit to the bulk liquid limit [48]. The $\sim 1-\mathrm{eV}$ blue shift of atomic states is the limiting value in the bulk environment. In the low-density surface region, the shift is expected to be much less. This qualitative picture has recently been confirmed for excited states of 25-atom helium clusters by the ab initio calculations of Closser and Head-Gordon [26]. Their analysis of cluster states that emerge from the atomic Rydberg $n=2$ manifold indicates that the ratio of bulk-state absorption to surface-state absorption increases with increasing energy across the absorption band centered at $\sim 21.6 \mathrm{eV}$. By translating these findings to the absorption band centered around $\sim 23.8 \mathrm{eV}$, a high ratio of surface-state excitations would be expected for energies below $\sim 24 \mathrm{eV}$, while above this en-

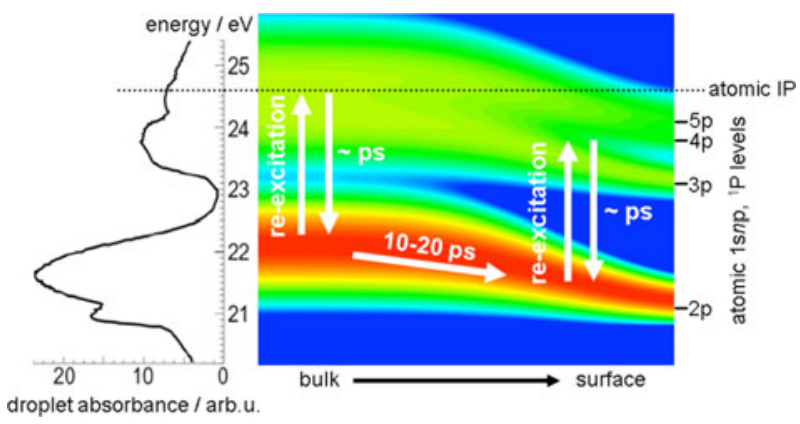

Fig. 9. Schematic of interband and intraband relaxation/re-excitation mechanisms in the bulk and surface regions of helium nanodroplets. A sequential relaxation model is proposed that transfers electronic excitation in the Rydberg $n=2$ manifold from the bulk region to the surface region within a time scale of $\sim 10-20 \mathrm{ps}$. See text for details.

ergy, perturbed 1s3p states (and other Rydberg states at higher energies) in the bulk become accessible.

For photon energies above the atomic IP, droplet ionization is often described by a multistep process initiated by an atomic ionization event that is followed by hole migration and eventually hole localization upon formation of an ionic dimer [23], [39]-[41]. Several authors have already pointed out that ionization near the cluster surface predominantly leads to the emission of dimer ions, while larger cluster fragments may be formed upon ionization in the cluster bulk [39], [41]. This trend is a consequence of an impulsive $\mathrm{He}_{2}{ }^{+}$ejection mechanism in combination with a reduced hole mobility in the low-densitysurface region [39], [41]. Due to the large average distance of helium atoms in the droplets (3.6 $\AA$ [35]), any newly formed dimer ions are highly vibrationally excited. Coupling of the dimer vibration into vibrational modes of the droplet is strongly disfavored due to the significant mismatch between the vibrational level spacings of the dimer ions and the low-frequency modes of the droplet. Cooling of the dimer vibrational motion and formation of stable dimer ions by evaporation of atoms from the droplet surface is, therefore, considered a minor process. Instead, bound dimer ions are probably formed in a series of 3- and 4-body collisions during which vibrational excitation is efficiently transformed into translational and rotational excitation [39], [40], eventually leading to the emission of ions from the droplet. If a dimer ion is originally formed in the droplet bulk, it may pick up more atoms on its way out, leading to the emission of larger fragment ions. In contrast, a dimer ion that is formed in the low-density surface region is most likely emitted from the droplet before significant fragment cluster growth sets in.

These findings can be directly applied to the pump-probe data presented here if one takes into account the intraband-interband relaxation scheme presented in our earlier publication [25] and that excited neutral atoms and molecules generated by the pump pulse are eventually expelled by the droplet in the form of Rydberg atoms and dimers [22].The following picture emerges (see Fig. 9). Excitation with the Sn-filtered pump beam leads predominantly to excitations in the surface region of the droplet. Ionization in this region of the droplet leads almost exclusively 
to dimer and trimer ion fragments as discussed by Callicoatt et al. [39] and Seong et al. [41], which is in agreement with the data in Figs. 3 and 5. For measurements with the Al filter, pump-pulse excitation of the droplet that does not lead to direct ionization generates atomic Rydberg states in both bulk $(>24 \mathrm{eV})$ and surface $(<24 \mathrm{eV})$ regions. If the probe pulse is incident before relaxation mechanisms have lowered the electronic energy of the excited states significantly, it can directly ionize the atoms. The location of the pump-probe-induced direct ionization within the droplet at small pump-probe time delays ( $<200 \mathrm{fs}$ ) is, therefore, directly related to the location of the initial excitation. In the case of the Al-filtered pump beam, this results in a significant contribution of ionized bulk states and consequently to the formation of larger ionic fragments. As interband relaxation to the lower electronic band $(\sim 21.6 \mathrm{eV})$ and/or migration of the excited atoms toward the droplet surface proceed, direct ionization by the probe pulse becomes energetically inaccessible. Instead, re-excitation into the upper electronic band below $24.6 \mathrm{eV}$ by the probe pulse leads to enhanced indirect ionization. As long as the probe pulse can re-excite the system to energies beyond $\sim 24 \mathrm{eV}$, indirect ionization will lead to small and large ionic fragments. However, once intraband relaxation and/or migration of excited atoms to the droplet surface have lowered the electronic energy below $\sim 22.4 \mathrm{eV}$, the probe pulse does not efficiently populate bulk states anymore, leading to a pump-probe ion spectrum that closely resembles the one recorded with the Sn-filtered pump beam. The data presented in Figs. 6 and 8 and the discussion in IV-B suggest that the relaxation to states below $\sim 22.4 \mathrm{eV}$ is completed after $\sim 10-20 \mathrm{ps}$.

Based on the simple model of density-dependent blue shifting of atomic states and supported by the calculations of Closser and Head-Gorden [26], the $\sim 10-20$ ps intraband relaxation in the lower electronic band is interpreted as a transfer of electronic excitation from bulk to surface states, i.e., as a "surfacing" process of bulk excitations that emerge from the $n=2$ Rydberg manifold. The physics of this excitation migration process is currently under investigation. Taking into account the average size of the droplets ( $\sim 40 \mathrm{~nm}$ diameter), the apparent mean velocity of the excitation migration is $\sim 1000-2000 \mathrm{~m} / \mathrm{s}$. This value exceeds estimated "bubble" migration speeds by about two orders of magnitude [34] and the speed of sound in bulk liquid helium at $0.4 \mathrm{~K}(\sim 240 \mathrm{~m} / \mathrm{s}$ [49]) by almost one order of magnitude. It is also significantly higher than the Landau critical velocity in bulk liquid helium $(\sim 60 \mathrm{~m} / \mathrm{s})$, which is commonly regarded as an upper limit for travel speeds of impurities in large ${ }^{4} \mathrm{He}$ clusters [34], [50]. The high apparent velocity may indicate that the excitation transport reported here is facilitated by a mechanism that has not yet been considered in related work on charge and energy transport in helium nanodroplets.

We note that the aforedescribed model does not readily provide an explanation for the high KEs of the ionic fragments that are assigned to ionization of bulk states. Transfer of vibrational into translational energy alone cannot account for the highest observed energies [51]. In the absence of significant vibrational excitation energies of the droplet environment, it is likely that the transfer of electronic into translational energy contributes to the observed KE distributions. This may be facilitated, for example, by repulsive interaction of the electronically excited neutral atoms with neighboring atoms and/or by release of energy gained in electrostrictive compression of the immediate environment of an ion inside a droplet. The former can, in principle, provide energies on the order of the maximum blue shift of atomic Rydberg states (1-1.6 eV [43]-[46]), the latter has been estimated by Buchenau et al. to provide up to $0.5-1.0 \mathrm{eV}$ [42]. Either of these processes may be accompanied by partial breakup of the original cluster prior to emission of the final, detected ionic fragments. The available data do not provide evidence for or against this scenario. The distinction, however, between surface and bulk excitation and ionization as the root cause for the observed differences between the Sn filter and the $\mathrm{Al}$ filter results is compatible with both alternatives. Note that earlier experimental studies have argued for an impulsive fragment ion-emission mechanism based solely on the absence of a clear signature of evaporative cooling [39]. In contrast, to the best of our knowledge, this is the first time that the KE provided by impulsive ion-emission mechanisms in pure helium droplets is directly quantified. We, therefore, hope that this study stimulates further theoretical work that is urgently needed to gain a deeper understanding of the relaxation and ionization dynamics in helium nanodroplets based on an extended set of available experimental data.

\section{Future Plans}

The ion measurements presented here clearly indicate strong variations in the relaxation and ionization dynamics of electronically excited droplets in the excitation energy range $23.25-24.75 \mathrm{eV}$. No distinction is made between different excitation energy regimes in [25], leading to the possibility that more relaxation channels may have contributed than originally anticipated. In order to gain a more detailed picture of the droplet de-excitation mechanisms, a new study is in preparation that concentrates on transient photoelectron spectra and transient ion KE distributions for excitation energies below $24 \mathrm{eV}$ only.

\section{CONCLUSION}

A novel ultrafast EUV-pump/IR-probe photoion-imaging technique is applied to monitor the relaxation dynamics of electronically excited helium nanodroplets. The structure and the dynamics of the mass spectra depend critically on the pump photon energy. Ion mass spectra recorded with pump photon energies above $\sim 24 \mathrm{eV}$ exhibit rich dynamics and differ significantly from ion mass spectra recorded at lower excitation energies.

The capability to record pump-probe time-resolved ion KE distributions proves to be instrumental to highlight different dynamic pathways in the complex relaxation scheme of the nanodroplets. Two major contributions to the de-excitation dynamics are identified: 1) A high KE component that is instantly populated within the time resolution of the experiment and that decays with the involvement of three timescales $(\sim 0.2-0.3 \mathrm{ps}$, $\sim 2-3 \mathrm{ps}$, and $\sim 10-20 \mathrm{ps}$ ) and 2) a low KE component that rises with the involvement of two time scales (few ps and $\sim 10-20 \mathrm{ps}$ ). The asymptotic trends of large and small ionic cluster yields for pump-probe time delays up to $\sim 100 \mathrm{ps}$ are complementary. 
A model is presented that explains the excitation-energydependent relaxation dynamics by differences between surface and bulk excitations. The model is supported by recent $a b$ initio calculations by Closser and Head-Gordon on 25 -atom clusters that indicate that surface and bulk states dominate lower and higher excitation energy ranges, respectively. An intraband relaxation mechanism in the lower electronic band of the droplets is proposed that proceeds on a $\sim 10-20$ ps-time scale and that corresponds to the transfer of electronic excitation from bulk to surface states in the Rydberg $n=2$ manifold.

\section{ACKNOWLEDGMENT}

The authors would like to thank C. Wang for providing unpublished ion mass spectra, K. Closser and M. Head-Gordon for stimulating discussions about the electronically excited states of helium nanodroplets.

\section{REFERENCES}

[1] L. Nugent-Glandorf, M. Scheer, D. A. Samuels, A. M. Mulhisen, E. R. Grant, X. Yang, V. M. Bierbaum, and S. R. Leone, "Ultrafast time-resolved soft X-ray photoelectron spectroscopy of dissociating Br2," Phys. Rev. Lett., vol. 87, no. 19, pp. 193002-1-193002-4, 2001.

[2] Ph. Wernet, M. Odelius, K. Godehusen, J. Gaudin, O. Schwarzkopf, and W. Eberhardt, "Real-time evolution of the valence electronic structure in a dissociating molecule," Phys. Rev. Lett., vol. 103, no. 1, pp. 013001-1013001-4, 2009

[3] Z.-H. Loh, M. Khalil, R. E. Correa, R. Santra, C. Buth, and S. R. Leone, "Quantum state-resolved probing of strong-field-ionized xenon atoms using femtosecond high-order harmonic transient absorption spectroscopy," Phys. Rev. Lett., vol. 98, no. 14, pp. 143601-1-143601-4, 2007.

[4] Z.-H. Loh and S. R. Leone, "Ultrafast strong-field dissociative ionization dynamics of $\mathrm{CH}_{2} \mathrm{Br}_{2}$ probed by femtosecond soft $\mathrm{x}$-ray transient absorption spectroscopy," J. Chem. Phys., vol. 128, no. 20, pp. 204302204308, 2008

[5] L. H. Haber, B. Doughty, and S. R. Leone, "Continuum phase shifts and partial cross sections for photoionization from excited states of atomic helium measured by high-order harmonic optical pump-probe velocity map imaging," Phys. Rev. A, Atom., Mol., Opt. Phys., vol. 79, no. 3, pp. 031401(R)-1-031401(R)-4, 2009.

[6] E. Gagnon, P. Ranitovic, X.-M. Tong, C. L. Cocke, M. M. Murnane, H. C. Kapteyn, and A. S. Sandhu, "Soft X-ray-driven femtosecond molecular dynamics," Science, vol. 317, no. 5843, pp. 1374-1378, 2007.

[7] A. S. Sandhu, E. Gagnon, R. Santra, V. Sharma, W. Li, P. Ho, P. Ranitovic, C. L. Cocke, M. M. Murnane, and H. C. Kapteyn, "Observing the creation of electronic feshbach resonances in soft X-ray-induced $\mathrm{O}_{2}$ dissociation," Science, vol. 322, no. 5904, pp. 1081-1085, 2008.

[8] J. v. Tilborg, T. K. Allison, T. W. Wright, M. P. Hertlein, R. W. Falcone, Y. Liu, H. Merdji, and A. Belkacem, "Femtosecond isomerization dynamics in the ethylene cation measured in an EUV-pump NIR-probe configuration," J. Phys. B, Atom., Mol. Opt. Phys., vol. 42, no. 8, pp. 081002-1081002-5, 2009.

[9] P. Antoine, E. Foumouo, B. Piraux, T. Shimizu, H. Hasegawa, Y. Nabekawa, and K. Midorikawa, "Two-photon double ionization of helium: An experimental lower bound of the total cross section," Phys. Rev. A, Atom., Mol. Opt.Phys.), vol. 78, no. 2, pp. 023415-1-023415-11, 2008.

[10] P. Ranitovic, X. M. Tong, B. Gramkow, S. De, B. DePaola, K. P. Singh, W. Cao, M. Magrakvelidze, D. Ray, I. Bocharova, H. Mashiko, A. Sandhu, E. Gagnon, M. M. Murnane, H. C. Kapteyn, I. Litvinyuk, and C. L. Cocke, "IR-assisted ionization of helium by attosecond extreme ultraviolet radiation," New J. Phys., vol. 12, no. 1, pp. 013008-1-013008-12, 2010.

[11] E. Gagnon, A. S. Sandhu, A. Paul, K. Hagen, A. Czasch, T. Jahnke, P. Ranitovic, C. L. Cocke, B. Walker, M. M. Murnane, and H. C. Kapteyn, "Time-resolved momentum imaging system for molecular dynamics studies using a tabletop ultrafast extreme-ultraviolet light source," Rev. Sci. Instrum., vol. 79, no. 6, p. 063102-063114, 2008.

[12] J. P. Toennies and A. F. Vilesov, "Superfluid helium droplets: A uniquely cold nanomatrix for molecules and molecular complexes," Angew. Chem. Int. Ed., vol. 43, no. 20, pp. 2622-2648, 2004.

[13] M. Wewer and F. Stienkemeier, "Molecular versus excitonic transitions in PTCDA dimers and oligomers studied by helium nanodroplet isolation spectroscopy," Phys. Rev. B, vol. 67, no. 12, pp. 125201-1-125201-5, 2003.

[14] V. Mozhayskiy, M. N. Slipchenko, V. K. Adamchuk, and A. F. Vilesov, "Use of helium nanodroplets for assembly, transport, and surface deposition of large molecular and atomic clusters," J. Chem. Phys., vol. 127, no. 9, pp. 094701-1-094701-6, 2007.

[15] K. Nauta and R. E. Miller, "Nonequilibrium self-assembly of long chains of polar molecules in superfluid helium," Science, vol. 283, no. 5409, pp. 1895-1897, 1999.

[16] R. Fröchtenicht, J. P. Toennies, and A. Vilesov, "High-resolution infrared spectroscopy of $\mathrm{SF}_{6}$ embedded in He clusters," Chem. Phys. Lett., vol. 229, no. 1-2, pp. 1-7, 1994.

[17] M. Hartmann, R. E. Miller, J. P. Toennies, and A. Vilesov, "Rotationally resolved spectroscopy of $\mathrm{SF}_{6}$ in liquid helium clusters: A molecular probe of cluster temperature," Phys. Rev. Lett., vol. 75, no. 8, pp. 1566-1569, 1995.

[18] S. Grebenev, J. P. Toennies, and A. F. Vilesov, "Superfluidity within a small helium-4 cluster: The microscopic andronikashvili experiment," Science, vol. 279, no. 5359, pp. 2083-2086, 1998.

[19] F. Bierau, P. Kupser, G. Meijer, and G. von Helden, "Catching proteins in liquid helium droplets," Phys. Rev. Lett., vol. 105, no. 13, pp. 133402-1$133402-4,2010$.

[20] J. C. H. Spence and R. B. Doak, "Single molecule diffraction," Phys. Rev. Lett., vol. 92, no. 19, pp. 198102-1-198102-4, 2004.

[21] M. Joppien, R. Karnbach, and T. Möller, "Electronic excitations in liquid helium: The evolution from small clusters to large droplets," Phys. Rev. Lett., vol. 71, no. 16, pp. 2654-2657, 1993.

[22] K. von Haeften, A. R. B. de Castro, M. Joppien, L. Moussavizadeh, R. von Pietrowski, and T. Möller, "Discrete visible luminescence of helium atoms and molecules desorbing from helium clusters: The role of electronic, vibrational, and rotational energy transfer," Phys. Rev. Lett., vol. 78, no. 23, pp. 4371-4374, 1997.

[23] R. Fröchtenicht, U. Henne, J. P. Toennies, A. Ding, M. Fieber-Erdmann, and T. Drewello, "The photoionization of large pure and doped helium droplets," J. Chem. Phys., vol. 104, no. 7, pp. 2548-2556, 1996.

[24] D. S. Peterka, A. Lindinger, L. Poisson, M. Ahmed, and D. M. Neumark, "Photoelectron imaging of helium droplets," Phys. Rev. Lett., vol. 91, no. 4, pp. 043401-1-043401-4, 2003.

[25] O. Kornilov, C. C. Wang, O. Bünermann, A. T. Healy, and M. Leonard, "Ultrafast dynamics in helium nanodroplets probed by femtosecond timeresolved EUV photoelectron imaging," J. Phys. Chem. A, vol. 114, no. 3, pp. 1437-1445, 2010.

[26] K. D. Closser and M. Head-Gordon, "Ab initio calculations on the electronically excited states of small helium clusters," J. Phys. Chem. A, vol. 114, no. 31, pp. 8023-8032, 2010.

[27] E. J. Takahashi, Y. Nabekawa, H. Mashiko, H. Hasegawa, A. Suda et al., "Generation of strong optical field in soft X-ray region by using highorder harmonics," IEEE J. Sel. Top. Quantum Electron., vol. 10, no. 6, pp. 1315-1328, Nov./Dec. 2004.

[28] M. Vidal-Dasilva, M. Fernández-Perea, J. A. Méndez, J. A. Aznárez, and J. I. Larruquert, "Electron-beam deposited boron coatings for the extreme ultraviolet," Appl. Opt., vol. 47, no. 16, pp. 2926-2930, 2008.

[29] O. Kornilov, R. Wilcox, and O. Gessner, "Nanograting-based compact vacuum ultraviolet spectrometer and beam profiler for in situ characterization of high-order harmonic generation light sources," Rev. Sci. Instrum., vol. 81, no. 6, pp. 063109-1-063109-8, 2010.

[30] D. S. Peterka, J. H. Kim, C. C. Wang, and D. M. Neumark, "Photoionization and Photofragmentation of $\mathrm{SF}_{6}$ in Helium Nanodroplets," J. Phys. Chem. B, vol. 110, no. 40, pp. 19945-19955, 2006.

[31] W. C. Wiley and I. H. McLaren, "Time-of-flight mass spectrometer with improved resolution," Rev. Sci. Instrum., vol. 26, no. 12, pp. 1150-1157, 1955.

[32] J. Ullrich, R. Moshammer, R. Dörner, O. Jagutzki, V. Mergel, H. SchmidtBocking, and L. Spielberger, "Recoil-ion momentum spectroscopy," J. Phys. B, Atom., Mol. Opt. Phys., vol. 30, no. 13, pp. 2917-2974, 1997.

[33] C. C. Wang, private communication, Oct. 2010.

[34] K. von Haeften, T. Laarmann, H. Wabnitz, and T. Möller, "Bubble formation and decay in $\mathrm{H}^{3} \mathrm{e}$ and $\mathrm{H}^{4} \mathrm{e}$ clusters," Phys. Rev. Lett., vol. 88, no. 23, pp. 233401-1-233401-4, 2002.

[35] D. S. Peterka, J. H. Kim, C. C. Wang, L. Poisson, and D. M. Neumark, "Photoionization dynamics in pure helium droplets," J. Phys. Chem. A, vol. 111, no. 31, pp. 7449-7459, 2007.

[36] C. Kim, S. Yurgenson, C. C. Hu, and J. A. Northby, "Photodetachment of metastable helium molecules from the surface of helium nanodroplets," J. Low Temp. Phys., vol. 113, pp. 1097-1102, 1998. 
[37] S. Yurgenson, C. C. Hu, C. Kim, and J. A. Northby, "Detachment of metastable helium molecules from helium nanodroplets," The Eur. Phys. J. D, vol. 9, no. 1, pp. 153-157, 2012.

[38] A. V. Benderskii, R. Zadoyan, N. Schwentner, and V. A. Apkarian, "Photodynamics in superfluid helium: Femtosecond laser-induced ionization, charge recombination, and preparation of molecular Rydberg states," $J$. Chem. Phys., vol. 110, no. 3, pp. 1542-1557, 1999.

[39] B. E. Callicoatt, K. Förde, L. F. Jung, T. Ruchti, and K. C. Janda, "Fragmentation of ionized liquid helium droplets: A new interpretation," $J$. Chem. Phys., vol. 109, no. 23, pp. 10195-10200, 1998.

[40] M. Ovchinnikov, B. L. Grigorenko, K. C. Janda, and V. A. Apkarian, "Charge localization and fragmentation dynamics of ionized helium clusters," J. Chem. Phys., vol. 108, no. 22, pp. 9351-9361, 1998.

[41] J. Seong, K. C. Janda, N. Halberstadt, and F. Spiegelmann, "Short-time charge motion in $\mathrm{He}_{n}^{+}$clusters," J. Chem. Phys., vol. 109, no. 24, pp. 10873-10884, 1998.

[42] H. Buchenau, J. P. Toennies, and J. A. Northby, "Excitation and ionization of ${ }^{4} \mathrm{He}$ clusters by electrons," J. Chem. Phys., vol. 95, no. 11, pp. 8134$8148,1991$.

[43] J. R. Broomall, W. D. Johnson, and D. G. Onn, "Density dependence of the electron surface barrier for fluid ${ }^{3} \mathrm{He}$ and ${ }^{4} \mathrm{He}, "$ Phys. Rev. B, vol. 14, no. 7, pp. 2819-2825, 1976.

[44] U. Asaf and I. T. Steinberger, "The energies of excess electrons in helium," Chem. Phys. Lett., vol. 128, no. 1, pp. 91-94, 1986.

[45] K. Martini, J. P. Toennies, and C. Winkler, "Electron scattering from ${ }^{4} \mathrm{He}$ and $\mathrm{Ne}$ clusters: determination of the cluster density from the electronic surface barrier potential," Chem. Phys. Lett., vol. 178, no. 4, pp. 429-434, 1991.

[46] U. Henne and J. P. Toennies, "Electron capture by large helium droplets," J. Chem. Phys., vol. 108, no. 22, pp. 9327-9338, 1998

[47] C. C. Wang, O. Kornilov, O. Gessner, J. H. Kim, D. S. Peterka, and D. M. Neumark, "Photoelectron imaging of helium droplets doped with Xe and Kr atoms," J. Phys. Chem. A, vol. 112, no. 39, pp. 9356-9365, 2008.

[48] J. Harms, J. P. Toennies, and F. Dalfovo, "Density of superfluid helium droplets," Phys. Rev. B, vol. 58, no. 6, pp. 3341-3350, 1998.

[49] W. M. Whitney and C. E. Chase, "Velocity of sound in liquid helium at low temperatures," Phys. Rev. Lett., vol. 9, no. 6, pp. 243-245, 1962.

[50] M. Schlesinger, M. Mudrich, F. Stienkemeier, and W. T. Strunz, "Dissipative vibrational wave packet dynamics of alkali dimers attached to helium nanodroplets," Chem. Phys. Lett., vol. 490, no. 4-6, pp. 245-248, 2010.

[51] P. J. Knowles and J. N. Murrell, "The structures and stabilities of helium cluster ions," Mol. Phys., vol. 87, no. 4, pp. 827-827, 1996.

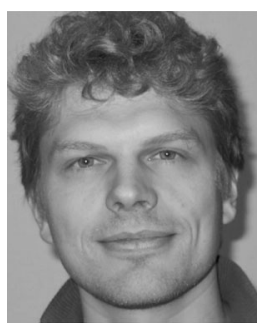

Oliver Bünermann was born in Lemgo, Germany, in 1978. He received the Dipl.-Phys. degree in fundamental physics in 2003, and the Dr.rer.nat. degree in experimental physics in 2006, both from the University of Bielefeld, Germany.

From 2006 to 2009, he was a Research Assistant in the group of Frank Stienkemeier at the University of Freiburg, Germany. Then he was a Postdoctoral Research Assistant (with D. Neumark, S. R. Leone, and O. Gessner) at Lawrence Berkeley National Laboratory for one year. He is currently a Postdoctoral Research assistant (with A. Wodtke) at the Georg-August-University, Göttingen, Germany.

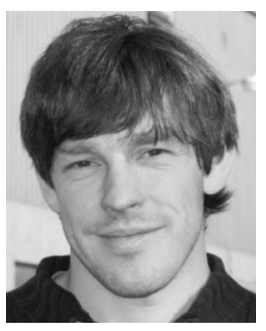

Oleg Kornilov was born in St. Petersburg, Russia, in 1978. He received the Magister in Physics degree in 2001 , and the Candidate degree in physical and mathematical sciences in 2004, both from St. Petersburg State University, St.Petersburg, Russia.

From 2001 to 2005, he was a Research Assistant in the group of Prof. J. Peter Toennies at the Max-Planck Institute for Flow Research, Germany. From 2006 to 2010, he was a Postdoctoral Fellow at the Chemical Sciences Division, Lawrence Berkeley Laboratory (in the groups of D. M. Neumark, S. R. Leone, and O. Gessner). He is currently a Staff Scientist at the Max-BornInstitute, Berlin, Germany.

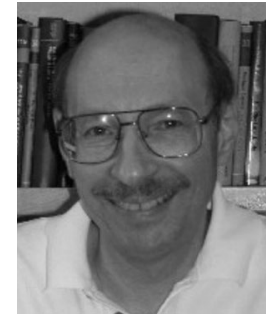

Stephen R. Leone was born in New York City, in 1948. He received the B.A. degree in chemistry from Northwestern University, Evanston, IL, in 1970, and the $\mathrm{Ph} . \mathrm{D}$. degree in chemistry from the University of California, Berkeley, in 1974

From 1974 to 1976, he was an Assistant Professor at University of Southern California. From 1976 to 2002, he was at JILA, the joint institute of University of Colorado and National Institute of Standards and Technology (NIST), where he became a Fellow of NIST and Adjoint Full Professor. Since 2002, he has been a Professor of Chemistry and Physics at the University of California, Berkeley, where he is also the Director of the Chemical Dynamics Beamline, Lawrence Berkeley National Laboratory.

Prof. Leone is a recipient of the American Chemical Society Pure Chemistry Award (1982), the American Physical Society Herbert P. Broida Prize (1989), and the American Chemical Society Peter Debye Award (2005). He is a member of the National Academy of Sciences, a Fellow of the American Academy of Arts and Sciences, a Fellow of the American Physical Society, and a Fellow of the Optical Society of America.

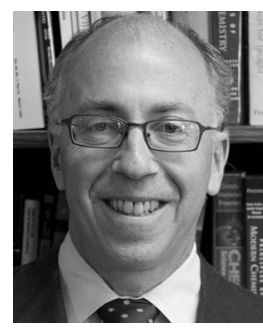

Daniel M. Neumark was born in Chicago, IL, in 1955. He received the B.A. degree in chemistry and physics from Harvard University, Cambridge, and the M.A. degree in chemistry in 1977, and the Ph.D. degree in physical chemistry from the University of California, Berkeley, in 1984

From 1984 to 1986, he was a Postdoctoral Fellow at JILA, the joint institute of University of Colorado and National Institute of Standards and Technology, and the University of Colorado. Since 1986, he has been a Professor of Chemistry at the University of California, Berkeley. He was the Director of the Chemical Sciences Division, Lawrence Berkeley National Laboratory during 2000-2010 and has been the Chair of the Department of Chemistry, since 2010.

Prof. Neumark is a recipient of the American Chemical Society Nobel Laureate Signature Award (2001), the Optical Society of America William F. Meggers Award (2005), and the American Chemical Society Irving Langmuir Award in Chemical Physics (2008). He is a Fellow of the American Physical Society, a Fellow of the American Association for the Advancement of Science, and a Fellow of the American Academy of Arts and Sciences.

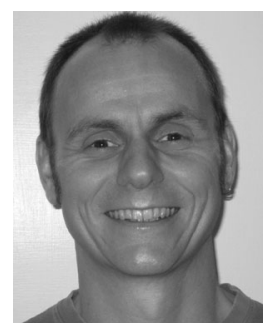

Oliver Gessner was born in Bonndorf/Schwarzwald, Germany, in 1969. He received the Dip.-Phys. degree in fundamental physics in 1996, and the Dr. rer. nat. degree in 2002 in experimental physics, both from the Technical University Berlin, Berlin, Germany.

He was a Canadian Government Laboratory Visiting Fellow during 2002-2005 and an Assistant Research Officer during 2005-2006 (with A. Stolow) at the Steacie Institute for Molecular Sciences of the National Research Council of Canada. Since 2006, he has been a Divisional Fellow in the Ultrafast X-ray Science Laboratory, Chemical Sciences Division, Lawrence Berkeley National Laboratory.

Dr. Gessner is a recipient of the Steacie Institute for Molecular Sciences Annual Award for Scientific Breakthrough and Technical Innovation (2005). 